\title{
CHITOSAN-MODIFIED COTTON THREAD FOR THE PRECONCENTRATION AND COLORIMETRIC TRACE DETERMINATION OF Co(II)
}

Willian Toito Suarez, ${ }^{1}$ Mathews O. K. Franco, ${ }^{1}$ Luis Fermín Capitán-Vallvey, ${ }^{2,3}$ Miguel M. Erenas ${ }^{2,3}$

${ }^{1}$ Department of Chemistry, Centre for Exact Sciences and Technology, 36570-900, Federal University of Viçosa, Viçosa - MG, Brazil. ${ }^{2}$ Department of Analytical Chemistry. ${ }^{3}$ Unit of Excellence in Chemistry applied to Biomedicine and the Environment, University of Granada. Campus Fuentenueva, Faculty of Sciences, 18071, University of Granada, Spain. 

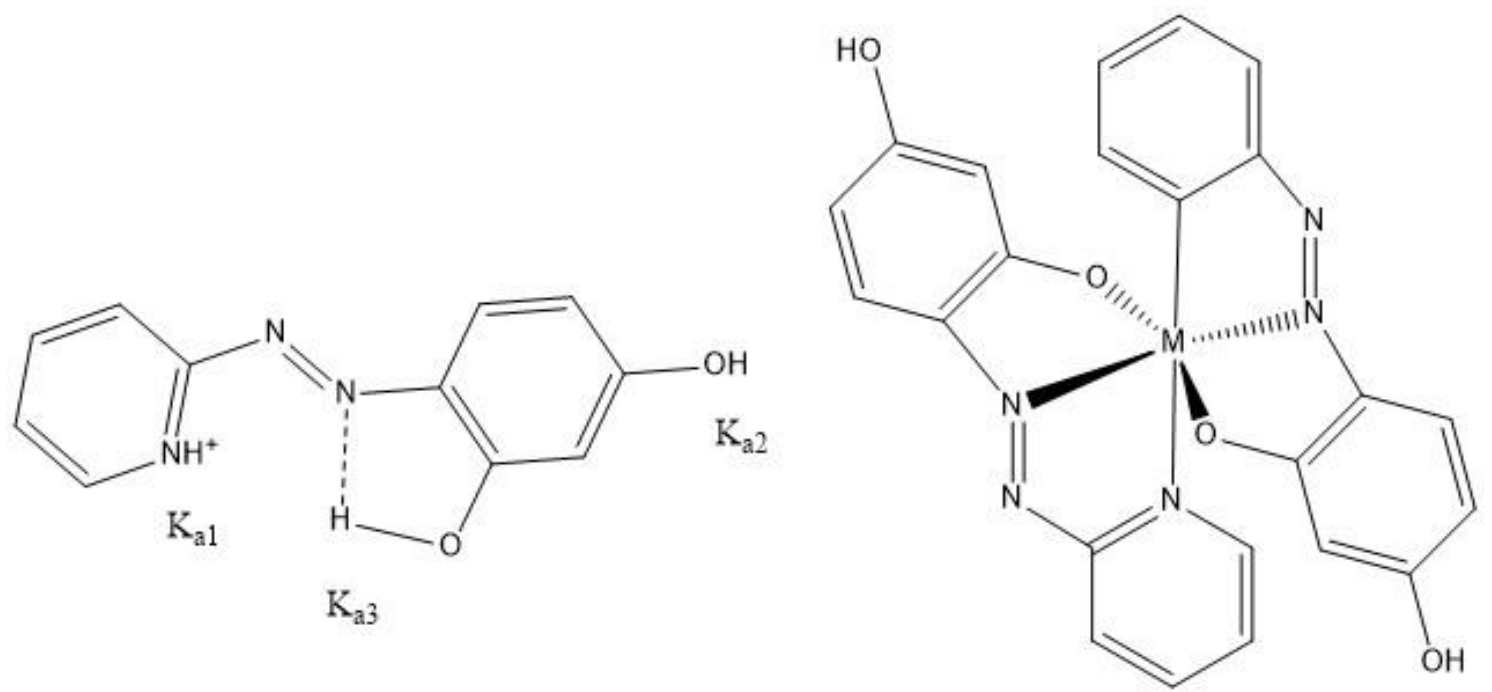

Figure S1. 4-(2-pyridylazo) resorcinol (PAR) molecule (left) and PAR complex (right). 


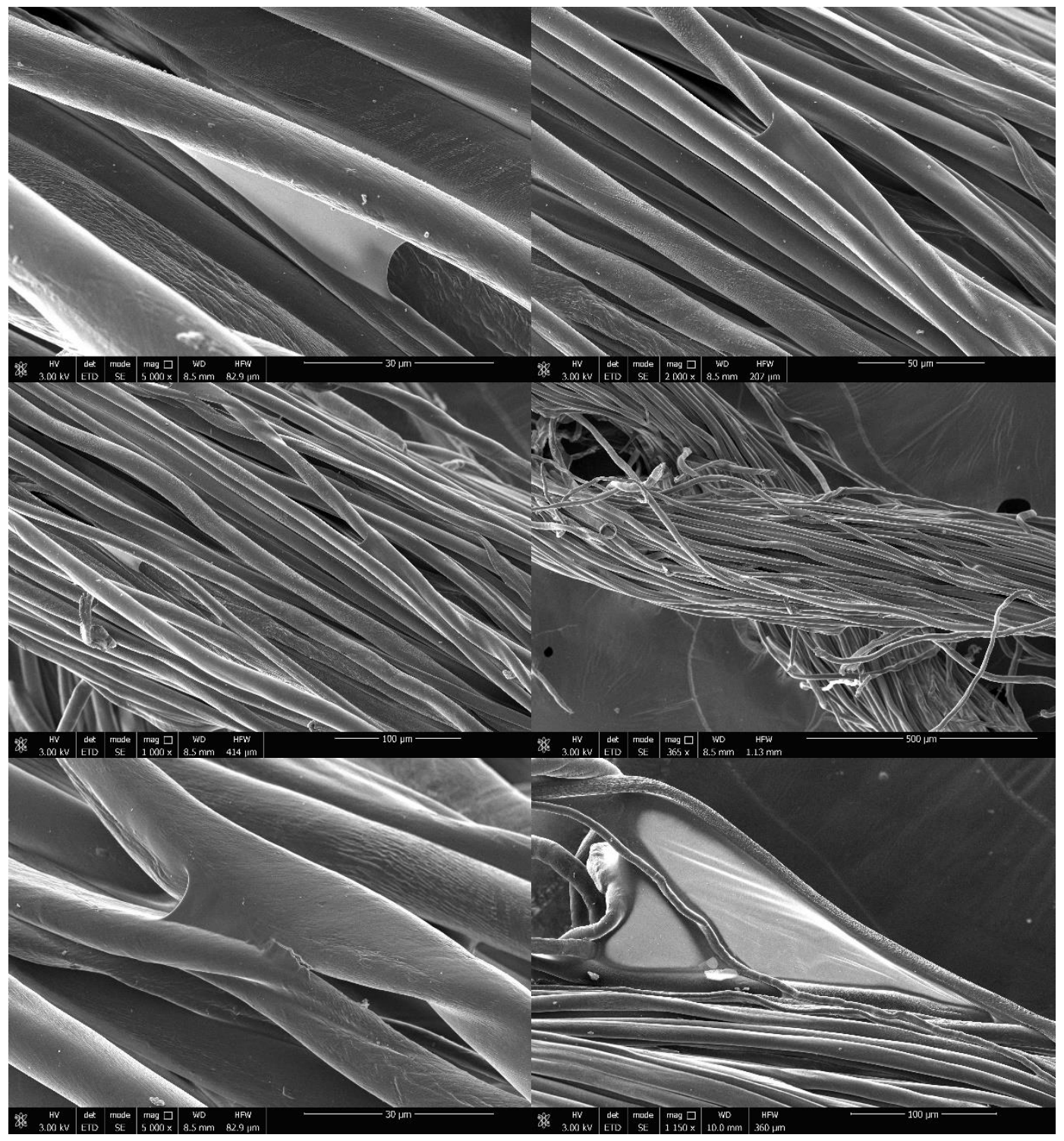

Figure S2. Different images of $\mu \mathrm{TAD}$ sensing zone containing chitosan. Obtained with FEG-ESEM, QuenScan 650F FEI@ electronic microscope together with an EverhartThornley detector (ETD), circular backscatter detector (CBS) and energy-dispersive detector (EDS). From Centre for Scientific Instrumentation, University of Granada, Spain. 


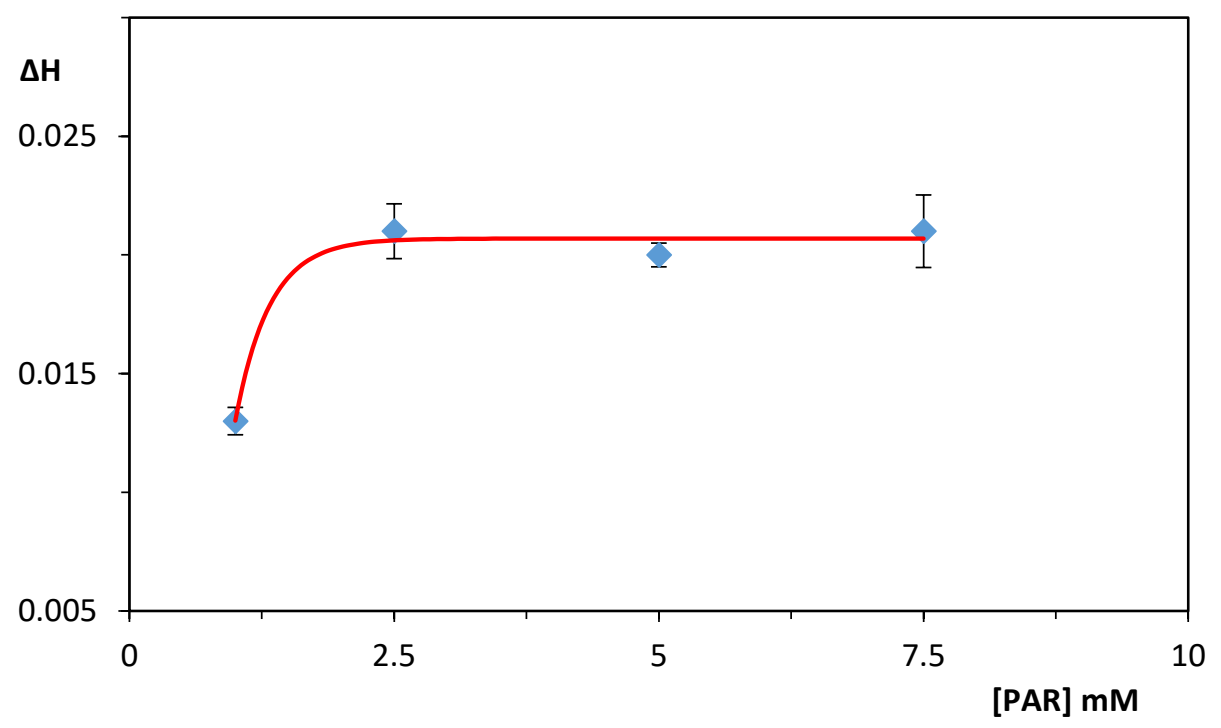

Figure S3. Study of the influence of PAR concentration on $\Delta \mathrm{H}\left(\mathrm{H}_{\mathrm{PAR}}-\mathrm{H}_{\mathrm{PAR}-\mathrm{Co}}\right)$.

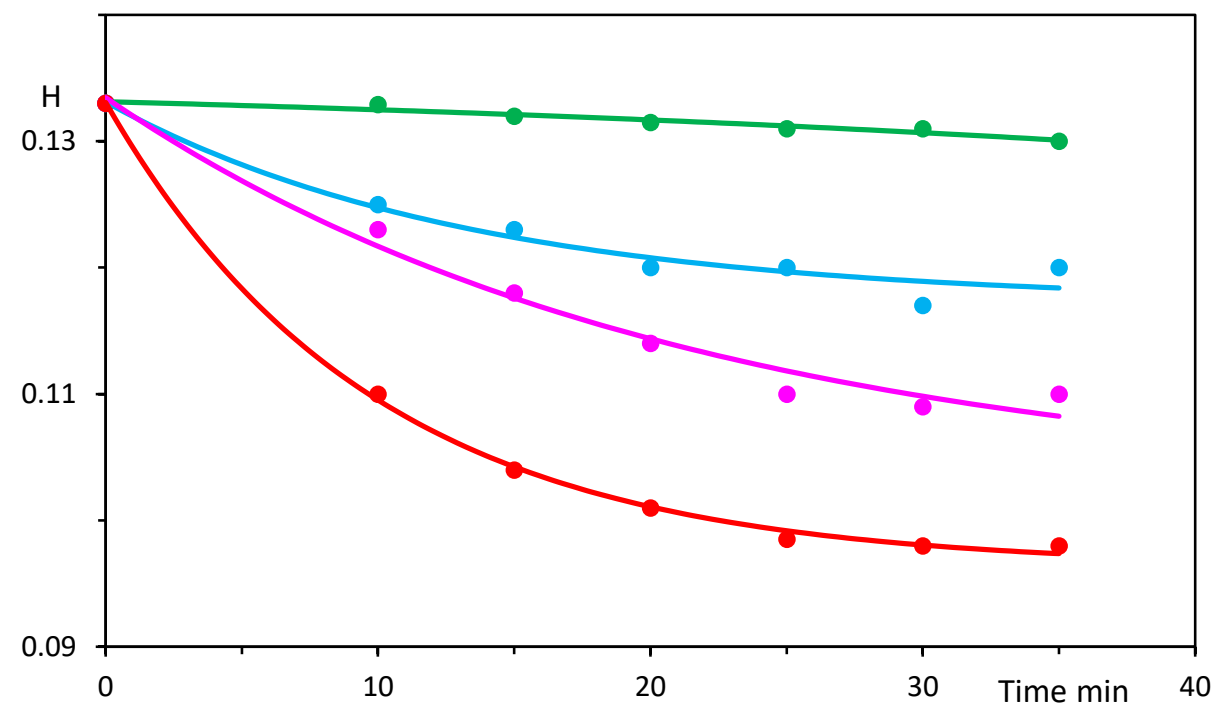

Figure S4. Evolution of $\mathrm{H}$ with time at different $\mathrm{Co}$ (II) concentrations: $50 \mu \mathrm{g} \cdot \mathrm{L}^{-1}$ (green data), $100 \mu \mathrm{g} \cdot \mathrm{L}^{-1}$ (blue data), $150 \mu \mathrm{g} \cdot \mathrm{L}^{-1}$ (magenta data) and $200 \mu \mathrm{g} \cdot \mathrm{L}^{-1}$ (red data). 
Table S1. Determination of Co(II) in spiked water samples using Atomic Absorption Spectrometry (AAS) as reference method $(n=3)$

\begin{tabular}{cccccc}
\hline Sample & $\begin{array}{c}\text { Found } \\
\left(\mu \mathbf{g} \cdot \mathbf{L}^{-1}\right)\end{array}$ & $\begin{array}{c}\text { Added } \\
\left(\mu \mathrm{g} \cdot \mathbf{L}^{-1}\right)\end{array}$ & $\begin{array}{c}\boldsymbol{\mu} \mathrm{TAD} \\
\left(\boldsymbol{\mu g} \cdot \mathbf{L}^{-1}\right)\end{array}$ & $\begin{array}{c}\text { AAS } \\
\left(\boldsymbol{\mu g} \cdot \mathbf{L}^{-1}\right)\end{array}$ & $\mid$ Error $\mid(\%)$ \\
\hline Tap water & $<6.5$ & 50 & $52 \pm 2$ & $49.2 \pm 0.3$ & 5.7 \\
& $<6.5$ & 100 & $99 \pm 2$ & $101.0 \pm 0.5$ & 2.0 \\
& $<6.5$ & 150 & $155 \pm 3$ & $152.0 \pm 0.1$ & 1.7 \\
& $<6.5$ & 200 & $208 \pm 2$ & $199.5 \pm 0.2$ & 4.3 \\
& $<6.5$ & 250 & $262 \pm 3$ & $248.9 \pm 0.2$ & 5.3 \\
& $<6.5$ & 300 & $288 \pm 3$ & $301.2 \pm 0.4$ & 4.4 \\
\hline
\end{tabular}

Table S2. Figures of merit of microfluidic devices for Co(II) determination in water.

\begin{tabular}{cccc}
\hline Substrate & Linear Range & LOD & Reference \\
\hline Paper & Qualitative & $50 \mu \mathrm{M}$ & {$[1]$} \\
\hline Paper & Semiquantitative & $0.5 \mu \mathrm{g} \cdot \mathrm{L}^{-1}$ & {$[2]$} \\
\hline Paper & $590-5.9 \times 10^{5} \mu \mathrm{g} \cdot \mathrm{L}^{-1}$ & $58.9 \mu \mathrm{g} \cdot \mathrm{L}^{-1}$ & {$[3]$} \\
\hline Paper & $500-2000 \mu \mathrm{g} \cdot \mathrm{L}^{-1}$ & $590 \mu \mathrm{g} \cdot \mathrm{L}^{-1}$ & {$[4]$} \\
\hline Paper & $10-1000 \mu \mathrm{M}$ & $1 \mu \mathrm{M}$ & {$[5]$} \\
\hline Thread & $25-600 \mu \mathrm{g} \cdot \mathrm{L}^{-1}$ & $6.50 \mu \mathrm{g} \cdot \mathrm{L}^{-1}$ & This work
\end{tabular}


[1] L. Feng, X. Li, H. Li, W. Yang, L. Chen, Y. Guan, Enhancement of sensitivity of paper-based sensor array for the identification of heavy-metal ions, Analytica Chimica Acta, 780 (2013) 74-80.

[2] L.H. Mujawar, A.A. Felemban, M.S. El-Shahawi, Hexamethyldisilazane modified paper as an ultra-sensitive platform for visual detection of $\mathrm{Hg}^{2+}, \mathrm{Co}^{2+}, \mathrm{Zn}^{2+}$ and the application to semi-quantitative determination of $\mathrm{Hg}^{2+}$ in wastewater, Analytical Sciences, 32 (2016) 491-497.

[3] M. Rahbar, P.N. Nesterenko, B. Paull, M. Macka, Geometrical Alignment of Multiple Fabrication Steps for Rapid Prototyping of Microfluidic Paper-Based Analytical Devices, Analytical Chemistry, 89 (2017) 11918-11923.

[4] K.R. Chabaud, J.L. Thomas, M.N. Torres, S. Oliveira, B.R. McCord, Simultaneous colorimetric detection of metallic salts contained in low explosives residue using a microfluidic paper-based analytical device ( $\mu$ PAD), Forensic Chemistry, 9 (2018) 35-41.

[5] L.H. Mujawar, M.S. El-Shahawi, Poly(methyl methacrylate)-modified cellulose fibers patterned with highly selective chromogenic reagent for rapid and trace determination of $\mathrm{Co}^{2+}$ in water, Analytical Methods, 10 (2018) 4454-4462. 